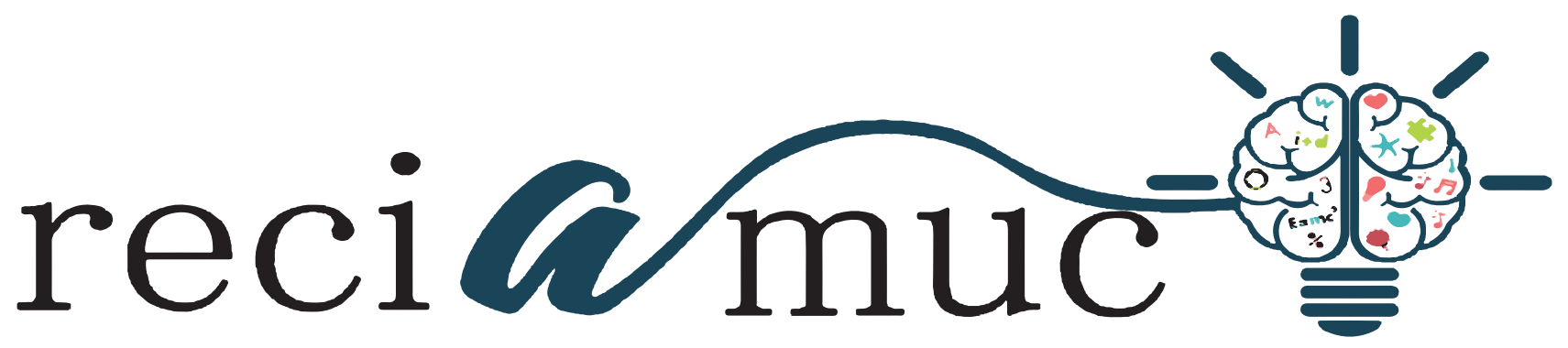

DOI: 10.26820/reciamuc/5.(4).noviembre.2021.129-138

URL: https://reciamuc.com/index.php/RECIAMUC/article/view/735

EDITORIAL: Saberes del Conocimiento

REVISTA: RECIAMUC

ISSN: 2588-0748

TIPO DE INVESTIGACIÓN: Artículo de Revisión

Código UNESCO: 32 Ciencias Médicas

PAGINAS: $129-138$

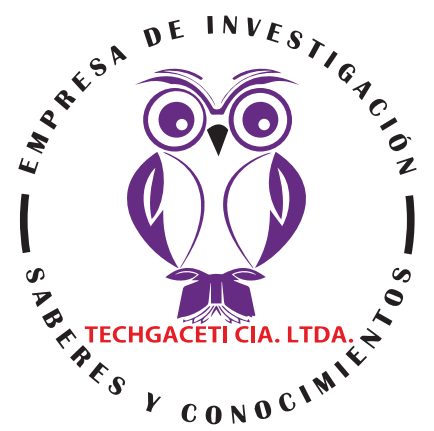

\title{
Carcinoma hepatocelular. Diagnóstico diferencial
} Hepatocellular carcinoma. Differential diagnosis Carcinoma hepatocelular. Diagnóstico diferencial

\section{Karla Abigail Navarrete Bolaños'; Jessica Cristina Andrade Villarreal2; Daysi Maricela Jacho Oña3; Jenifer Lizbeth Calapaqui Araque ${ }^{4}$}

\section{RECIBIDO: 15/09/2021 ACEPTADO: 05/10/2021 PUBLICADO: 29/11/2021}

1. Médico General; Investigador Independiente; Ambato, Ecuador; karlita_navarreteb@hotmail.com; (D) https:// orcid.org/0000-0002-4365-160X

2. Médico General; Investigador Independiente; Tulcán, Ecuador; andradejessica1994@gmail.com; (D) https:// orcid.org/0000-0002-2929-807X

3. Médico General; Investigador Independiente; Latacunga, Ecuador; jachodaysi@gmail.com; (D) https://orcid. org/0000-0002-2398-6920

4. Médico General; Investigador Independiente; Quito, Ecuador; issaliz0109@gmail.com; (D) https://orcid. org/0000-0002-2262-6288

\section{CORRESPONDENCIA}

Karla Abigail Navarrete Bolaños

karlita_navarreteb@hotmail.com

Ambato, Ecuador

๑ RECIAMUC; Editorial Saberes del Conocimiento, 2021 


\section{RESUMEN}

El carcinoma hepatocelular o hepatocarcinoma es la neoplasia primaria hepática maligna más común a nivel mundial. Por lo general se desarrolla en pacientes con enfermedad hepática crónica que han desarrollado una cirrosis de cualquier etiología, más es particularmente asociada con a con infección por los virus de la hepatitis B y C (VHB y VHC). El objetivo de este estudio, elaborado bajo un diseño documental y enmarcado a una metodología de revisión, se centra en el abordaje de varios aspectos del carcinoma heptocelular (CHC), y en especial de su diagnóstico diferencial. En los resultados se exponen generalidades, diagnóstico diferencial de carcinoma hepatocelular y pronóstico de la misma. Se concluye que, diagnóstico diferencial de CHC en adultos y niños, puede consistir en hiperplasia nodular focal (HNF) y adenoma hepático; aunque más propiamente en adultos serían las lesiones focales en el hígado, tumores metastásicos y carcinoma de las vías biliares intrahepáticas, mientras que en niños se destaca primordialmente el hepatoblastoma, el sarcoma embrionario indiferenciado, y otras neoplasias hepáticas malignas menos comunes.

Palabras clave: Metastásicos, cáncer, hepatocito, cirrosis, hepatitis.

\section{ABSTRACT}

Hepatocellular carcinoma or hepatocarcinoma is the most common primary malignant hepatic neoplasm worldwide. It usually develops in patients with chronic liver disease who have developed cirrhosis of any etiology, but it is particularly associated with infection with hepatitis $\mathrm{B}$ and $\mathrm{C}$ viruses (HBV and HCV). The objective of this study, prepared under a documentary design and framed by a review methodology, focuses on addressing various aspects of heptocellular carcinoma (HCC), and especially its differential diagnosis. The results show generalities, differential diagnosis of hepatocellular carcinoma and its prognosis. It is concluded that the differential diagnosis of HCC in adults and children may consist of focal nodular hyperplasia (UFH) and hepatic adenoma; although more properly in adults it would be focal lesions in the liver, metastatic tumors and carcinoma of the intrahepatic bile ducts, while in children hepatoblastoma, undifferentiated embryonal sarcoma, and other less common malignant liver neoplasms stand out.

Keywords: Metastatic, cancer, hepatocyte, cirrhosis, hepatitis.

\section{RESUMO}

O carcinoma hepatocelular ou hepatocarcinoma é a neoplasia hepática maligna primária mais comum em todo o mundo. Geralmente se desenvolve em pacientes com doença hepática crônica que desenvolveram cirrose de qualquer etiologia, mas está particularmente associada à infecção pelos vírus da hepatite B e C (HBV e HCV). O objetivo deste estudo, elaborado sob um desenho documental e enquadrado por uma metodologia de revisão, centra-se em abordar diversos aspectos do carcinoma heptocelular (CHC), e principalmente seu diagnóstico diferencial. Os resultados mostram generalidades, diagnóstico diferencial do carcinoma hepatocelular e seu prognóstico. Conclui-se que o diagnóstico diferencial de $\mathrm{CHC}$ em adultos e crianças pode consistir em hiperplasia nodular focal (HNF) e adenoma hepático; embora mais apropriadamente em adultos seriam lesões focais no fígado, tumores metastáticos e carcinoma das vias biliares intra-hepáticas, enquanto em crianças se destacam hepatoblastoma, sarcoma embrionário indiferenciado e outras neoplasias hepáticas malignas menos comuns.

Palavras-chave: Metastático, câncer, hepatócito, cirrose, hepatite. 


\section{Introducción}

El carcinoma hepatocelular $(\mathrm{CHC})$ o hepatocarcinoma (HCC), es el tipo más común de cáncer primario de hígado, originado en el principal tipo de célula hepática (hepatocito). Este tipo de patología mayormente evoluciona en pacientes que padecen enfermedades hepáticas crónicas, tales como como: las infecciones con el virus de la hepatitis $\mathrm{B}(\mathrm{VHB})$ o virus de la hepatitis $\mathrm{C}$ (VHC), la cirrosis. Otras de las condiciones que también incrementan dichos riesgos son: el hígado que ha cicatrizado luego de infecciones con $\mathrm{VHB}$ o $\mathrm{VHC}$, el consumo excesivo de bebidas alcohólicas, acumulación de grasa en el hígado. (Mayo Foundation for Medical Education and Research - MFMER, 2019)

Los signos y síntomas, suelen ser inespecíficos y el diagnóstico se apoya en las pruebas de diagnóstico por la imagen, en las concentraciones de alfa-fetoproteína (AFP) $\mathrm{y}$, ocasionalmente, en la biopsia hepática. (Tholey, 2020)

Reig et al. (2020), en cuanto al criterio diagnóstico señalaron que, luego de que en 2016 se editaran las guías del manejo del carcinoma hepatocelular $(\mathrm{CHC})$, han surgido nuevas propuestas, las cuales responden a los cambios epidemiológicos relacionados con la curación del virus de la hepatitis $\mathrm{C}$ y el aumento de otros factores de riesgo, como el síndrome metabólico asociado con la enfermedad hepática por depósito de grasa. Esto igualmente ha incidido en nuevas recomendaciones para la indicación de tratamientos quirúrgicos que han sido adoptadas particularmente por entes como: las asociaciones americana (AASLD) y europea (EASL) para el estudio de la enfermedades del hígado o por las distintas sociedades científicas involucradas en el manejo del CHC. Es así como entonces surgen nuevos retos en el manejo clínico de pacientes que padecen esta seria condición.
Por otra parte, en el Manual Medicina Interna Basada en la Evidencia 2019/20, se deja ver que el criterio diagnóstico esta basado en lo que demuestren las pruebas de imagen de tomografía computada (TC) o resonancia magnética nuclear (RMN), cuando la lesión sea $>2 \mathrm{~cm}$, y en caso contrario, será necesario un examen histológico (con una imagen adecuada en la TC o en la RMN.

En el sumario del PDQ® del Instituto $\mathrm{Na-}$ cional del Cáncer ( $\mathrm{NCl}$, por sus siglas en inglés), de información sobre exámenes de detección del cáncer de hígado (hepatocelular) se describen, en breve, los siguientes datos sobre la incidencia, mortalidad y factores de riesgo asociada al $\mathrm{CHC}$ :

En 2020, el cáncer de hígado fue el sexto cáncer más frecuente y la tercera causa principal de muerte por cáncer en el mundo.

En todo el mundo, las hepatitis B y C crónicas se reconocen como los factores principales que aumentan el riesgo de $\mathrm{CHC}$; el riesgo aumenta cuando hay coinfección por el virus de la hepatitis $\mathrm{B}$ y el virus de la hepatitis C. La incidencia de $\mathrm{CHC}$ en las personas con hepatitis crónica alcanza $0,46 \%$ al año. [...] La cirrosis también es un factor de riesgo de $\mathrm{CHC}$, cualquiera sea su origen. El riesgo anual de $\mathrm{CHC}$ en las personas con cirrosis oscila entre 1 y $6 \%$. Otros factores de riesgo son la cirrosis alcohólica, la hemocromatosis, la deficiencia de $\alpha$-1-antitripsina, las glucogenosis, la porfiria cutánea tardía, la tirosinemia y la enfermedad de Wilson, además de la cirrosis biliar, aunque esta última es infrecuente. [...] En algunas partes de África, es posible que la incidencia alta de $\mathrm{CHC}$ en los humanos se vincule con la ingesta de alimentos contaminados con aflatoxinas. Sin embargo, esta relación se confunde por la coexistencia frecuente de la infección por hepatitis $B$ en esos grupos de población. (Consejo Editorial del $P D Q \AA$ de información sobre el cáncer, 2021a) 
En otra sinopsis PDQ®; que igualmente fue desarrollada por los anteriores expertos pero en fecha distinta, y que trata sobre la prevención del CHC se mencionan de manera concisa algunos datos en relación a la incidencia, mortalidad y supervivencia:

A nivel mundial, el cáncer de hígado es el sexto tipo de cáncer más frecuente y la tercera causa principal de mortalidad por cáncer. En todo el mundo, cada año se diagnostican 905677 casos nuevos de $\mathrm{CHC}$ y se producen 830180 muertes por esta enfermedad; en la mayoría de los países, las tasas anuales de incidencia y mortalidad de $\mathrm{CHC}$ son casi idénticas. El CHC es el quinto diagnóstico de cáncer más frecuente en varones y el noveno diagnóstico de cáncer más frecuente en mujeres. La incidencia del CHC varía mucho según la ubicación geográfica. Las regiones de incidencia más alta son el norte y oeste de África (Egipto, Gambia, Guinea) y el este o sureste de Asia (Mongolia, Camboya y Vietnam). La incidencia de $\mathrm{CHC}$ es baja en América del Norte y América del Sur, la mayor parte de Europa, Australia y partes del Oriente Medio. En todo el mundo, el CHC es más frecuente en hombres que en mujeres. (Consejo Editorial del PDQ® de información sobre el cáncer, 2021b)

El objetivo de este estudio, elaborado bajo un diseño documental y enmarcado a una metodología de revisión, se centra en el abordaje de varios aspectos del carcinoma heptocelular $(\mathrm{CHC})$, y en especial de su diagnóstico diferencial, a fines de proporcionar un material actualizado que sea útil no solo para la adquisición y el fortalecimiento del conocimiento sino también como fundamento futuras investigaciones.

\section{Materiales y Métodos}

El presente trabajo de investigación, enmarcado en una metodología de revisión, está orientado a la construcción de un material bibliográfico actualizado, enfocado en abordar el tema de carcinoma hepatocelular y diagnósticos diferenciales.
Entre las bases de datos consultadas destacan: Organización Panamericana de la Salud (PAHO), Organización Mundial de la Salud (OMS), Biblioteca Virtual de la Salud (BVS), Pubmed, entre otras.

La búsqueda se fundamentó en el uso de expresiones propias, constituidas en base a palabras clave, términos específicos y operadores lógicos y booleanos. Los mejores resultados se obtuvieron con las siguientes formulaciones: carcinoma hepatocelular AND diagnostico diferencial; y hepatocarcinoma + diagnostico diferencial. A dichos resultados se les aplicaron criterios de refinamiento tales como: idioma español e inglés; publicación entre 2011 y 2021; área investigativa en salud y medicina humana; tipo de bibliografía: artículos originales (papers) en revistas científicas y académicas; boletines, noticias o resúmenes informativos de estudios clínicos en humanos, ensayos controlados aleatorizados, estudios casos y controles, series de casos, estudios de cohortes, estudios prospectivos de cohortes, reportes de casos, material de conferencias o ponencias; tesis de grado, posgrado y doctorado; guías de práctica clínica, protocolos o manuales de procedimientos, monografías de expertos y cualquier otro documento que mostrase información de interés con fundamento científico

De igual manera fueron adelantados otras sondeos menores sin considerar la aplicación de cualquier otro criterio de descarte, ya que se requirió encontrar información complementaria que independientemente de su origen o época de publicación, es considerada igualmente importante ajustada y de relevancia para este tema.

Fue luego de los anteriores procesos que, entonces, se prosiguió con la lectura crítica y análisis interpretativo de toda información definitivamente seleccionada para de allí exponer las ideas tratadas como evidencia investigativa. 


\section{Resultados}

El carcinoma hepatocelular $(\mathrm{CHC})$ es la neoplasia primaria hepática maligna más común a nivel mundial. Por lo general se desarrolla en pacientes con enfermedad hepática crónica que han desarrollado una cirrosis de cualquier etiología, más es particularmente asociada con a con infección por los virus de la hepatitis B y C (VHB y VHC). (López, 2015; Forner et al., 2016) Este último, particularmente ha reconocido que recientemente han aparecido novedades importantes en el diagnóstico, estadificación y tratamiento del $\mathrm{CHC}$.

El CHC es un problema médico relevante. Actualmente constituye la sexta neoplasia más frecuente en el mundo y la tercera causa de muerte por cáncer. Su distribución mundial es muy heterogénea y está estrechamente relacionada con la prevalencia variable de los diferentes factores de riesgo asociados al desarrollo de esta enfermedad. La incidencia es máxima en el Sudeste asiático y África Subsahariana. La mayor parte de los casos en esa zona se hallan en relación con el virus de la hepatitis $B(\mathrm{VHB})$, asociado o no a aflatoxina, y la incidencia excede los 15 casos/100.000 hab/año. El sur de Europa, incluyendo España, presenta una incidencia intermedia, de 5-10 casos/100.000 hab/año, y finalmente, el norte de Europa y América tienen la menor incidencia, aproximadamente de 5 casos/100.000 hab/año. En ambas zonas desempeñan un papel predominante la infección por virus de la hepatitis C (VHC) y el alcoholismo. No obstante, en los últimos años se han evidenciado ciertos cambios epidemiológicos en diversas áreas. (Forner et al., 2016)

En los niños, también se considera un tumor hepático poco frecuente, agresivo y maligno, que se origina en los hepatocitos. Por lo general, la evolución de ésta patología en dicho grupo cursa a partir de los 10 años de edad, en un contexto no cirrótico en el $70 \%$ de los casos, sin embargo, también puede ocurrir, con menor frecuencia, en uno cirrótico.

Las neoplasias malignas hepáticas primarias son poco frecuentes en niños y en adolescentes. El carcinoma hepatocelular ( $\mathrm{CHC}$ ) constituye menos del $25 \%$ de las mismas. La incidencia anual es de 1/2.000.000 aproximadamente, con unas tasas de incidencia más elevadas en África subsahariana y en el sudeste asiático como resultado de la hepatitis B endémica (VHB) y de la exposición a aflatoxinas. El CHC aparece con mayor frecuencia en varones (2-3:1) siendo la incidencia más elevada en adolescentes (1/1.250.000).

El $\mathrm{CHC}$ se desarrolla principalmente en pacientes pediátricos sin una enfermedad hepática subyacente a diferencia de los adultos, en los que por lo general está asociado a cirrosis hepática por la ingesta de alcohol. El CHC pediátrico se desarrolla principalmente en niños y adolescentes y, excepcionalmente, antes de los 5 años de edad. Las principales manifestaciones de presentación son masa abdominal acompañada de dolor, inflamación y molestias, así como pérdida de peso y anorexia. Con menor frecuencia, se observa esplenomegalia, náuseas, vómitos e ictericia. Las metástasis en los ganglios linfáticos mediastínicos, pulmones, cerebro y médula ósea es frecuente en los estadios avanzados de la enfermedad (en un 25\% de casos en el momento del diagnóstico). El CHC puede estar asociado a enfermedades congénitas, tales como la tirosinemia, la atresia biliar, la tirosinemia tipo 1 (en el $50 \%$ de los casos), la deficiencia de alfa-1 antitripsina, la colestasis intrahepática familiar progresiva, los síndromes de Alagille o de Gardner, la enfermedad de Wilson o de almacenamiento de glucógeno I-IV, la anemia de Fanconi, la poliposis adenomatosa familiar (PAF), la hiperplasia nodular focal y la hemocromatosis. (Orphanet, 2019)

Reig et al. (2020), al destacar aspectos clave en la confirmación del diagnóstico $\mathrm{CHC}$ 
(Figura 1) han dicho que; es viable sin necesidad de confirmación patológica, cuando en el paciente con hepatopatía crónica le es detectado mediante un estudio dinámico de imagen (TC o RM) un nódulo mayor o igual a $1 \mathrm{~cm}$ de tamaño que muestra una intensa captación de contraste en fase arterial seguida de lavado en fase portal (y/o venosa si se trata de una TC o de una RM con contraste extracelular). En caso de que no sea típico el patrón vascular de la lesión, éste se basará en la biopsia.

Por último, también expusieron que, si el nódulo es $<1 \mathrm{~cm}$, se aconseja llevar un riguroso seguimiento ecográfico cada 3-4 meses a fines de descubrir un eventual incremento de tamaño, dado que en estos casos, la probabilidad de que sea de naturaleza maligna es baja, aparte de la dificultad que supone su educada identificación. Lo anterior la finalidad de detectar su posible crecimiento, para entonces aplicar los criterios diagnósticos antes referidos (Reig, et al; 2020)

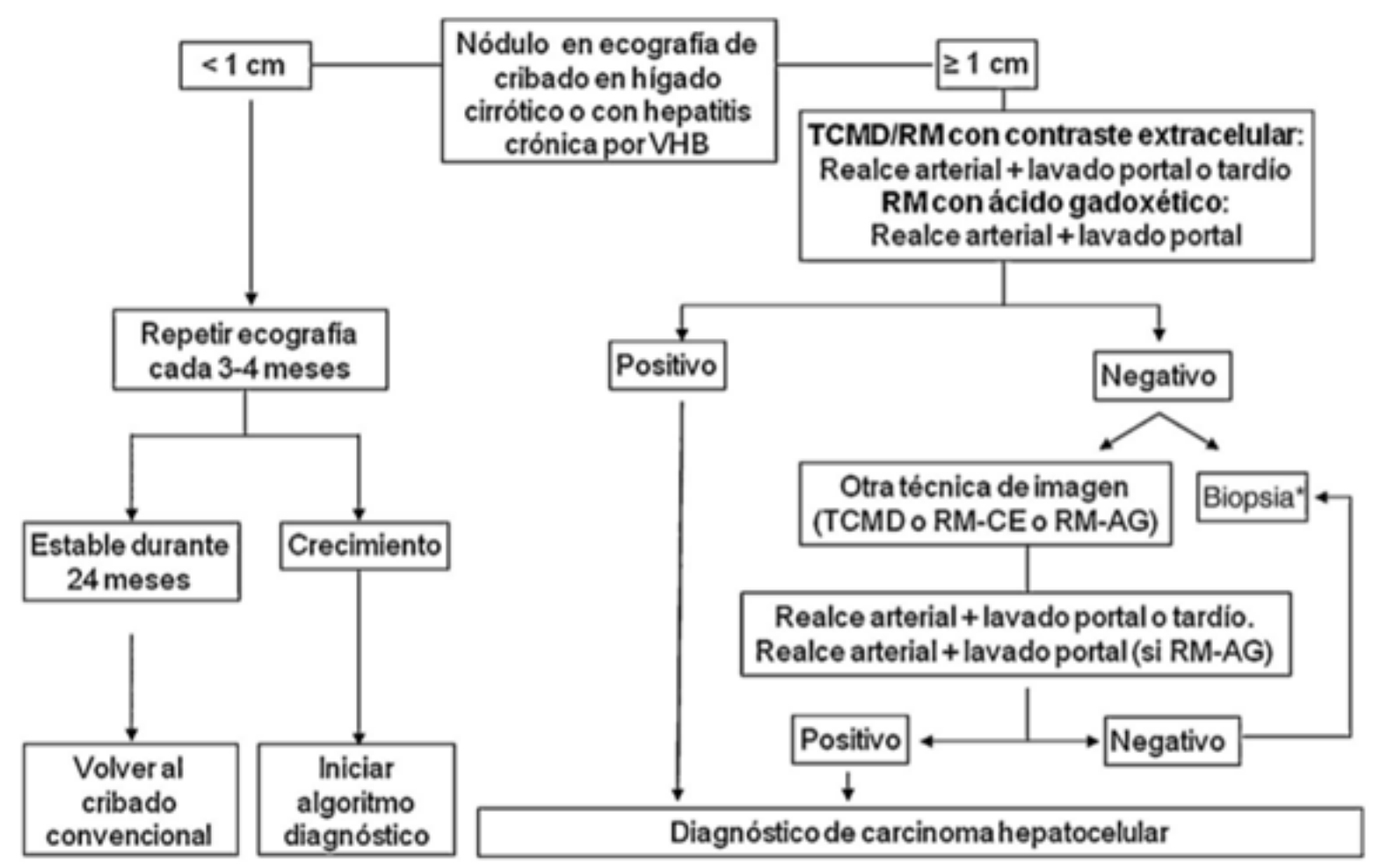

Figura 1. Algoritmo para el diagnóstico de carcinoma hepatocelular.

Fuente: TCMD: TC multidetector, RM-CE: Resonancia magnética con contraste extraceular, RM-AG: Resonancia magnética con ácido gadoxético.

** Dado que la probabilidad obtener un resultado falso negativo puede llegar hasta en un $30 \%$ en nódulos menores de $2 \mathrm{~cm}$, en caso de biopsia negativa, considerar repetirla o seguimiento estrecho por imagen

Tomada de; Diagnóstico y tratamiento del carcinoma hepatocelular. Actualización del documento de consenso de la AEEH, AEC, SEOM, SERAM, SERVEI y SETH. Reig et al. (2020). P.7. https://www.sciencedirect.com/science/article/pii/S0025775320307697?via\%3Dihub 
En la mayoría de los pacientes se puede realizar el diagnóstico adecuado por las características de las imágenes (en general, las lesiones $<1,0 \mathrm{~cm}$. son benignas). A los fines diagnósticos, los nódulos hepáticos se diferencian entre los que ocurren en pacientes con cirrosis y pacientes sin cirrosis. Hasta que se demuestre lo contrario, un tumor hepático en un hígado cirrótico debe ser considerado un HC; los tumores hepáticos múltiples en un hígado cirrótico indican $\mathrm{HC}$ difus o, en raras ocasiones, los nódulos con un grado elevado de displasia, un linfoma hepático. [...] En más del 20\% de la población en general se hallan lesiones hepáticas benignas, incluyendo el angioma (4\%), la hiperplasia nodular focal $(0,4 \%)$ y adenomas hepáticos (0,004\%). Las lesiones hepáticas múltiples en un hígado normal por lo general indican un hígado metastático (el más común, del adenocarcinoma, colon, estómago, pulmón o próstata), pero podrían ser quistes o hemangiomas. Es raro hallar metástasis hepáticas en un hígado cirótico. Las lesiones hepáticas múltiples de naturaleza benigna como los hemangiomas o la hiperplasia nodular focal, no son infrecuentes en un hígado normal. (Papponetti, 2012) (Tabla 1)

\section{Diagnóstico diferencial del $\mathrm{CHC}$}

Conforme al Manual de Medicina Interna Basada en la Evidencia 2019/20, el diagnóstico diferencial para esta patología se basa en la detección de lesiones focales en el hígado, entre las cuales pueden figurar: adenoma hepático, tumores metastásicos, carcinoma de las vías biliares intrahepáticas, hiperplasia nodular focal.

En la población pediátrica el diagnóstico diferencial que destaca es el hepatoblastoma, considerado como el principal tumor hepático de este grupo etario en países occidentales. No obstante, al igual que en la población adulta, la diferenciación diagnostica también podría consistir en la determinación de hiperplasia nodular focal (HNF), el adenoma hepático y otras neoplasias hepáticas malignas menos comunes, y se agrega el sarcoma embrionario indiferenciado. (Orphanet, 2019)

En el estudio colaborativo de Bañales et al. (2017); que estuvo centrado en la búsqueda de nuevos biomarcadores no invasivos para el diagnóstico del colangiocarcinoma (CCA), el carcinoma hepatocelular (HCC) y la colangitis esclerosante primaria (PSC); se expuso que, hasta el momento de ese estudio no se habían definidos biomarcadores precisos por métodos no invasivos que facilitaran el diagnóstico diferencial de dichas patologías, de hecho, sugerían que, hay casos en los que es difícil el diagnóstico diferencial entre colangiocarcinoma y carcinoma hepatocelular.

Otro criterio importante de mencionar en este aspecto es el que se ha encontrado en Leendertz (2021)

La capacidad de detección del CHC en un hígado cirrótico presenta dificultades debido a la presencia de nódulos regenerativos y displásicos, alteraciones en la perfusión hepática y la presencia de otras neoplasias hepáticas.

Para establecer un diagnóstico diferencial del CHC por TC y RM se han establecido varias recomendaciones destinados a la evaluación del hígado cirrótico o a la hepatitis B crónica sin cirrosis, los cuales con el tiempo se han integrado en el LI-RADS de la ACR (American College of Radiology) (pág. 27)

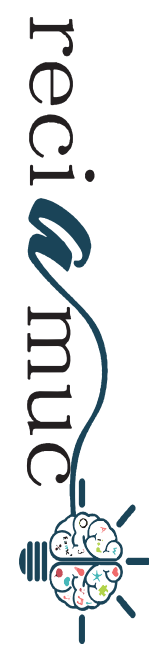


Tabla 1. Diagnóstico clínico diferencial de la mayoría de los tumores hepáticos.

\begin{tabular}{|c|c|c|c|c|}
\hline & Hígado cirrótico & $\begin{array}{l}\text { Lesiones } \\
\text { comunes }\end{array}$ & Hígado no cirrótico & $\begin{array}{l}\text { Lesiones } \\
\text { comunes }\end{array}$ \\
\hline $\begin{array}{l}\text { Tumores } \\
\text { malignos }\end{array}$ & $\begin{array}{l}\text { Carcinoma hepatocelular } \\
\text { Colangiocarcinoma } \\
\text { Nódulo hiperplásico de alto } \\
\text { grado } \\
\text { Linfoma } \\
\text { Metástasis (excepcional) }\end{array}$ & a.d & $\begin{array}{c}\text { Metástasis } \\
\text { HC bien diferenciado } \\
\text { HC fibrolamelar } \\
\text { Colangiocarcinoma } \\
\text { Hemangioendotelioma } \\
\text { Linfoma } \\
\text { Melanoma } \\
\text { Tumor neuroendocrino } \\
\text { Sarcoma(angiosarco-ma, } \\
\text { leiomiosarcoma) }\end{array}$ & $\begin{array}{c}\text { a.b } \\
\text { a.b.c.g }\end{array}$ \\
\hline $\begin{array}{l}\text { Tumores } \\
\text { benignos }\end{array}$ & $\begin{array}{l}\text { Displasia de bajo grado } \\
\text { Hígado graso focal } \\
\text { Hemangioma } \\
\text { Adenoma hepático }\end{array}$ & $\mathrm{g}$ & $\begin{array}{c}\text { Hemangioma Hiperplasia } \\
\text { nodular focal } \\
\text { Adenoma hepático } \\
\text { Hiperplasia regenerativa } \\
\text { nodular } \\
\text { Transformación nodular } \\
\text { parcial } \\
\text { Infiltración grasa focal } \\
\text { Adenoma del conducto } \\
\text { biliar }\end{array}$ & $\begin{array}{l}\mathrm{b} \\
\mathrm{a}, \mathrm{b} \\
\mathrm{a}, \mathrm{b} \\
\mathrm{b}, \mathrm{f} \\
\mathrm{e}, \mathrm{f} \\
\mathrm{c}, \mathrm{e}\end{array}$ \\
\hline
\end{tabular}

\section{Prevención del CHC y recomendaciones}

López (2015) y Garrido (2018) coinciden en señalar que la prevención de la aparición de un $\mathrm{CHC}$ es útil la inoculación contra el VHB, el tratamiento contra la hepatitis C, las campañas enfocadas en el mejoramiento de las condiciones sociosanitarias de la población (evitando la contaminación alimentaria por aflatoxina), y la promoción de hábitos de vida saludables.

La replicación del VHB se ha relacionado con un incremento del riesgo de $\mathrm{CHC}$, por lo que se sugiere que su tratamiento debería disminuir el desarrollo de este tumor.

Hay que tener en cuenta que una vez se establece la cirrosis hepática, a pesar de eliminar el factor etiológico, el riesgo de aparición de $\mathrm{CHC}$ continúa. Ya se ha expuesto que la mayoría de los pacientes con $\mathrm{CHC}$ están afectos de cirrosis. Además, cuanto más temprano sea el diagnóstico del tumor, más favorable será el pronóstico al detectarlo en estadios más precoces. (Garrido, 2018, pág. 37)

El uso de la vacuna contra HBV con el tiempo disminuye la incidencia, en especial en áreas endémicas. La prevención del desarrollo de cirrosis de cualquier origen ( $p$. ej., a través del tratamiento de la hepatitis C crónica, la detección temprana de la hemocromatosis, o el manejo del alcoholismo) también puede influir en forma significativa sobre la incidencia de cáncer. (Tholey, 2020)

Con base en lo expuesto por Reig et al. (2020), las recomendaciones son:

- La vacunación universal contra el VHB reduce la incidencia de $\mathrm{CHC}$ (evidencia alta, recomendación fuerte).

- En pacientes con hepatitis crónica viral se recomienda el tratamiento antiviral ya que ha demostrado su impacto en pre- 
venir la evolución a cirrosis y, por tanto, prevenir el desarrollo de $\mathrm{CHC}$ (evidencia alta, recomendación fuerte).

- Una vez establecida la lesión hepática asociada a riesgo clínicamente relevante (cirrosis, o incluso sin alcanzar la cirrosis en pacientes con infección por el VHB), la eliminación del agente etiológico disminuye, pero no elimina, el riesgo de aparición de CHC (evidencia alta, recomendación fuerte).

- Los pacientes con cirrosis asociada a $\mathrm{VHC}$ mantienen tanto el riesgo de $\mathrm{CHC}$ de novo como de recurrencia de $\mathrm{CHC}$, incluso después de alcanzar respuesta viral sostenida. Se recomienda mantener la estrategia de vigilancia convencional en estos pacientes (evidencia moderada, recomendación fuerte).

- Se ha demostrado que el consumo de café disminuye el riesgo de $\mathrm{CHC}$ en pacientes con enfermedad hepática crónica (evidencia moderada, recomendación fuerte).

- Los pacientes afectos de cirrosis hepática de cualquier etiología deben ser considerados para participar en programas de cribado (evidencia moderada, recomendación fuerte).

- La técnica de cribado más adecuada es la ecografía abdominal realizada por personal experto (evidencia moderada, recomendación fuerte).

- No se recomienda el uso de AFP como técnica de cribado (evidencia moderada, recomendación débil).

- La ecografía abdominal de cribado se debe realizar cada seis meses. El intervalo de cribado no necesita ser acortado en pacientes con mayor riesgo de desarrollar $\mathrm{CHC}$ (evidencia moderada, recomendación débil).

- No existen datos para poder realizar una recomendación en pacientes con enfermedad hepática por hígado graso (EHGNA) sin cirrosis y en pacientes VHC sin fibrosis avanzada que han conseguido RVS (evidencia baja, recomendación débil). (Reig et al., 2020, págs. 3-4)

\section{Pronostico del CHC}

Según Tholey (2020) cuando CHC se encuentra en un estado avanzado o si la función de síntesis hepática es atípica, se considera que el pronóstico es negativo, sin embargo, si el caso se trata de un pequeño tumor en el hígado, las terapias ablativas son paliativas y la resección quirúrgica o el trasplante de hígado en ocasiones son curativos.

En el área pediátrica, si la tumoración es resecable, el pronóstico se supedita al porcentaje de éxito de dicho procedimiento quirúrgico.

La tasa de supervivencia a 5 años es moderada en los tumores en estadios tempranos, pero estas tasas se reducen al $20-30 \%$ en pacientes con enfermedad avanzada. (Orphanet, 2019)

\section{Conclusión}

Sobre la base de las fuentes utilizadas en esta investigación es posible atreverse a deducir que, los expertos sostienen que, después de los tumores metastásicos o secundarios, el $\mathrm{CHC}$ consiste en el tumor primario maligno más frecuente en el hígado, particularmente en pacientes cirróticos de cualquier etiología o que padecen enfermedades hepáticas crónicas (VHB, VHC).

En cuanto al diagnóstico diferencial de $\mathrm{CHC}$ se encontró que, en adultos y niños, puede tratarse de hiperplasia nodular focal (HNF) y adenoma hepático; aunque más propiamente en adultos sería lesiones focales en el hígado, tumores metastásicos y carcinoma de las vías biliares intrahepáticas, mientras que en niños se destaca primordialmente el hepatoblastoma, el sarcoma embrionario indiferenciado, y otras neoplasias hepáticas malignas menos comunes.

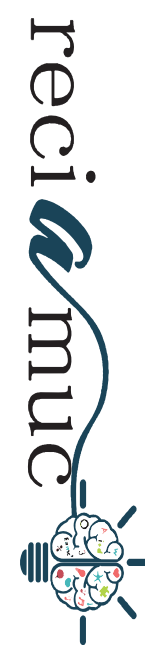




\section{Bibliografía}

Armas, R., \& Gajewski, P. (Edits.). (3a Ed). Manual de Medicina Interna Basada en la Evidencia 2019/20, On-Line. Recuperado el 10 de noviembre de 2021, de empendium.com: https://empendium.com/manualmibe/compendio/chapter/B34.II.7.16.4.

Bañales, J., Arbelaiz, A., Azkargorta, M., Krawczyk, M., Santos-Laso, A., Lapitz, A., . . . Lammert, F. (octubre de 2017). Serum extracellular vesicles contain protein biomarkers for primary sclerosing cholangitis and cholangiocarcinoma. Hepatology, 66(4), 1125-43. Recuperado el 10 de noviembre de 2021, de https://aasldpubs.onlinelibrary.wiley. com/doi/pdf/10.1002/hep.29291

Consejo Editorial del PDQ $®$ de información sobre el cáncer. (24 de septiembre de 2021a). PDQ Exámenes de detección del cáncer de hígado (hepatocelular). Recuperado el 10 de noviembre de 2021, de https://www.cancer.gov/espanol/tipos/ higado/pro/deteccion-higado-pdq\#_AboutThis_1

Consejo Editorial del PDQ® de información sobre el cáncer. (30 de julio de 2021b). PDQ Prevención del cáncer de hígado (hepatocelular). Recuperado el 10 de noviembre de 2021, de National Cancer Institute [NCl-NIH] (USA): https://www.cancer.gov/ espanol/tipos/higado/pro/prevencion-higado-pdq

Forner, A., Reig, M., Varela, M., Burrel, M., Feliu, J., Briceño, J., . . Bruix, J. (2016). Diagnóstico y tratamiento del carcinoma hepatocelular. Actualización del documento de consenso de la AEEH, SEOM, SERAM, SERVEI y SETH. Medicina Clínica, 146(11), e1-e22. doi:10.1016/j.medcli.2016.01.028
López, R. (2015). Neoplasias hepáticas malignas: 1a. parte. Hepatocarcinoma: papel de la biopsia hepática, estudios de inmunohistoquímica y otros aspectos importantes. Revista s Colombiana de Gastroenterología, 30(2), 232-242. Recuperado el 10 de noviembre de 2021, de http://www.scielo. org.co/pdf/rcg/v30n2/v30n2a14.pdf

Mayo Foundation for Medical Education and Research - MFMER. (04 de mayo de 2019). Cáncer de hígado. Recuperado el 10 de noviembre de 2021, de Mayo Clinic: https://www.mayoclinic.org/ es-es/diseases-conditions/hepatocellular-carcino$\mathrm{ma} / \mathrm{cdc}-20354552$

Orphanet. (octubre de 2019). BusquedalEnfermedadlCarcinoma hepatocelular pediátrico: Orphanet, 5.50.0. (P. Czauderna, \& M. Murawski, Editores) Recuperado el 10 de noviembre de 2021, de orpha.net: https://www.orpha.net/consor/cgi-bin/ OC_Exp.php?Expert=33402\&lng $=E S$

Reig, M., Forner, A., Ávila, M., Ayuso, C., Mínguez, B., Varela, M., ... Burrel, M. (7 de mayo de 2020). Diagnóstico y tratamiento del carcinoma hepatocelular. Actualización del documento de consenso de la AEEH, AEC, SEOM, SERAM, SERVEI y SETH [In Press]. Medicin Clínica, 156(9), 30 p. doi:10.1016/j.medcli.2020.09.022

Tholey, D. (abril de 2020). Carcinoma Hepatocelular. Recuperado el 10 de noviembre de 2021, de msdmanuals.com/ [en español]: https://www. msdmanuals.com/es-ve/professional/trastornos-hep\%C3\%A1ticos-y-biliares/tumores-y-granulomas-hep\%C3\%A1ticos/carcinoma-hepatocelular?query=Carcinoma\%20hepatocelular

\section{CITAR ESTE ARTICULO:}

Navarrete Bolaños, K. A., Andrade Villarreal, J. C., Jacho Oña, D. M., \& Calapaqui Araque, J. L. (2021). Carcinoma hepatocelular. Diagnóstico diferencial. RECIAMUC, 5(4), 129-138. https://doi.org/10.26820/reciamuc/5.(4).noviembre.2021.129-138

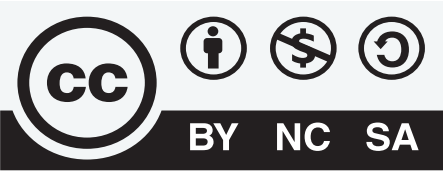

CREATIVE COMMONS RECONOCIMIENTO-NOCOMERCIAL-COMPARTIRIGUAL 4.0. 